\title{
Modulation of Gut Microbiota, Short-Chain Fatty Acid Production, and Inflammatory Cytokine Expression in the Cecum of Porcine Deltacoronavirus-Infected Chicks
}

OPEN ACCESS

Edited by: Ahmed Sayed Abdel-Moneim, Taif University, Saudi Arabia

Reviewed by: Salama Shany, Beni-Suef University, Egypt Samy Kasem, Kafrelsheikh University, Egypt

*Correspondence:

Zhan-yong Wei weizhanyong@henau.edu.cn

Specialty section: This article was submitted to

Virology,

a section of the journal

Frontiers in Microbiology

Received: 21 December 2019 Accepted: 16 April 2020

Published: 04 June 2020

Citation:

$L i H$, Zhang $H$, zhao F, Wang $S$, Wang $Z$ and Wei $Z$ (2020) Modulation of Gut Microbiota, Short-Chain Fatty Acid Production, and Inflammatory Cytokine Expression in the Cecum

of Porcine Deltacoronavirus-Infected Chicks. Front. Microbiol. 11:897. doi: 10.3389/fmicb.2020.00897

\author{
Hai-yan Li ${ }^{1}$, Hong-lei Zhang ${ }^{1,2}$, Fu-jie zhao ${ }^{1,2}$, Shi-qiong Wang ${ }^{1}$, Zhi-xiang Wang ${ }^{1}$ and \\ Zhan-yong Weit,2* \\ ${ }^{1}$ College of Animal Science and Veterinary Medicine, Henan Agricultural University, Zhengzhou, China, ${ }^{2}$ Key Laboratory \\ for Animal-Derived Food Safety of Henan Province, Henan Agricultural University, Zhengzhou, China
}

Porcine deltacoronavirus (PDCoV) is a novel swine enteropathogenic coronavirus that causes watery diarrhea and induces proinflammatory cytokine responses in piglets. Our previous research showed that the specific-pathogen-free (SPF) chicks exhibited mild diarrhea and low fecal viral shedding, along with cecum lesions after PDCoV infection. Disturbances in the homeostasis of the gut microbiota have been associated with various diseases. We aimed to explore the effects of PDCoV infection on chick gut microbiota, short-chain fatty acid (SCFAs) production, and inflammatory cytokine expression in chicks, and also to investigate the relationship between gut microbiota and SCFAs or inflammatory cytokine expression of the PDCoV-infected chicks. Results obtained using $16 \mathrm{~S}$ rRNA sequencing showed that infection with PDCoV strain HNZK02 significantly altered the composition of chick gut microbiota, with the reduced abundance of Eisenbergiella and Anaerotruncus genera at 5 days post-inoculation (dpi) $(P<0.05)$, and an increased abundance of Alistipes genus at $17 \mathrm{dpi}(P<0.05)$. The production of SCFAs in the cecum of PDCoV HNZK-02-infected chicks, including acetic acid, propionic acid, and butyric acid, decreased in all cases. The expression of inflammatory cytokines (interferon- $\gamma$, tumor necrosis factor- $\alpha$, and interleukin-10) was increased in the cecum tissue and serum of the PDCoV HNZK-02-infected chicks when detected by quantitative real-time polymerase chain reaction and enzyme-linked immunosorbent assay, respectively. Further analysis showed significant correlation between bacterial genera and SCFAs or inflammatory cytokines expression in cecum of the PDCoV infected chicks. These findings might provide new insight into the pathology and physiology of PDCoV in chicks.

Keywords: Porcine deltacoronavirus (PDCoV), SPF chick, intestinal microbiota, inflammatory cytokines, shortchain fatty acids

\footnotetext{
Abbreviations: AA, acetic acid; BA, butyric acid; dpi, days post inoculation; ELISA, enzyme-linked immunosorbent assay; GC, gas chromatography; Gn, gnotobiotic; IBD, infectious bursal disease; IFN- $\gamma$, interferon gamma; IL-10, interleukin 10; LDA, linear discriminant analysis; LEFSe, linear discriminant analysis effect size; LLC-PK, LLC-porcine kidney; OTUs, operational taxonomic units; PA, propionic acid; PDCoV, porcine deltacoronavirus; PCoA, principal coordinate analysis; SPF, the specific pathogen free; SCFAs, short-chain fatty acid; TNF- $\alpha$, tumor necrosis factor alpha.
} 


\section{INTRODUCTION}

Porcine deltacoronavirus (PDCoV) is an enveloped, positivesense single-stranded RNA virus, with a genome length of approximately $25 \mathrm{~kb}$ (Zhang, 2016). This virus was originally found in Hong Kong in 2012 (Woo et al., 2012), and the first outbreak of PDCoV-related diarrhea in swine herds was reported in the United States in 2014 (Wang et al., 2014). As an important enteropathogen in pigs, PDCoV can cause acute diarrhea, vomiting, and dehydration in neonatal piglets (Chen et al., 2014; $\mathrm{Hu}$ et al., 2016). Moreover, the infected pigs are characterized by thin and transparent intestinal walls and accumulation of large amounts of yellow fluid in the intestinal lumen (Jung et al., 2015; Li et al., 2019). Further, PDCoV-infected piglets show signs of proinflammatory cytokine responses during acute infection (Jung et al., 2018). PDCoV has caused huge economic losses for the pig industries. Apart from infection of gnotobiotic $(\mathrm{Gn})$ and conventional pigs, PDCoV was also reported to have limited ability to infect Gn calves (Jung et al., 2017). Recent studies showed the susceptibility of specific-pathogen-free (SPF) chicks to PDCoV infection, with the clinical syndrome of mild diarrhea and slight lesions in cecum, which was weaker than that of piglets infected with PDCoV (Liang et al., 2019; Boley et al., 2020). However, the reason why PDCoV infection can cause intestinal damage is still unknown.

The chicken gastrointestinal tract is an important site for immune cell development, which not only regulates gut microbiota, but also maintains extraintestinal immunity. Intestinal commensal microbes play a crucial role in gut homeostasis through mutually beneficial interactions with the host immune system (Deriu et al., 2016). Once the intestinal mucosal barrier and microbiota are destroyed, intestinal inflammation will occur. In turn, the host's inflammatory response will elicit changes in the microbial community structure (Deriu et al., 2016). The gut microbiota produces various metabolites, including short-chain fatty acids (SCFAs), which can regulate the host antiviral immune response (Budden et al., 2017). Propionate and butyrate have also been reported to induce differentiation of T-regulatory cells, assisting in control of intestinal inflammation (Donohoe et al., 2014; Louis et al., 2014).

In chicks, recent studies have emphasized the key roles of intestinal microbiota in shaping immunity against viral diseases, including avian influenza, Marek's disease, Newcastle disease (ND), and infectious bursal disease (IBD). These studies not only reflected connections between gut microbiota and distal organs in regulatory functions, but also emphasized the interaction between intestinal microbiota and viral infections and their impacts on immune regulations (Lillehoj and Trout, 1996; Balamurugan and Kataria, 2006; Li, 2007; Chen et al., 2017; Yitbarek et al., 2018). It was reported that different commensal bacteria had their own unique role against viral infection by modulating diverse immune mechanisms (Abt et al., 2012). In the H9N2 avian influenza virus infected chicks, increased levels of interferon gamma (IFN- $\gamma$ ). tumor necrosis factor alpha (TNF$\alpha$ ), and interleukin-17A (IL-17A) led to intestinal microflora dysbiosis (Li H. et al., 2018). However, there are no studies emphasizing how PDCoV affects the gut microbiota in chicks.
Thus, in the current study we aimed to observe the intestinal microbiota, SCFAs, and inflammation responses to PDCoV infection in chicks and elucidate the potential associations among them, which will provide the theoretical basis for investigation of the etiology and pathogenesis of PDCoV.

\section{MATERIALS AND METHODS}

\section{Virus}

The virulent PDCoV strain-HNZK-02 (GenBank accession number MH708123) was isolated and identified by our laboratory and propagated in LLC-porcine kidney (LLC-PK) cells. Passage 5 of this strain was used in current study. Virus propagation and virus titers were performed as described previously (Liang et al., 2019).

\section{Experimental Infection of SPF Chicks With PDCoV Strain-HNZK-02}

The SPF chicks were purchased from Jinan SIPAFAS Poultry Co. Ltd. in Jinan, China. Twenty-four-day-old SPF chicks with similar body weight (about $50 \mathrm{~g} / \mathrm{chick}$ ) were randomly divided into two groups, the control (mock) and PDCoV HNZK-02infected groups. All chicks were housed in the same facility but in different rooms under biosafety conditions and allowed free access to water and feed during the experiment. Feed was formulated to meet or exceed the National Research Council nutrient requirements for chicks (National Research Council [NRC], 1994; Table 1). Each chick was intragastrically inoculated with PDCoV strain HNZK-02 or Eagle's Minimum Essential Medium (MEM) with $300 \mu \mathrm{L} /$ chick (10.5 $\log 10 \mathrm{GE} /$ chick) ( $n=10 /$ per group).

\section{Sample and Tissue Collection}

Based on our previous study (Liang et al., 2019), three chicks in each group were selected randomly for necropsy at 5 and $17 \mathrm{dpi}$, respectively. The cecal contents were collected for analysis of

TABLE 1 | The composition and nutrients of the basal diet.

\begin{tabular}{lclc}
\hline Ingredient & Content (\%) & Chemical composition & Content \\
\hline Corn & 55 & CP,\% & 20.6 \\
Soybean meal & 31 & ME, Mcal/kg & 3 \\
Wheat shorts & 3.3 & Calcium,\% & 1 \\
Fish meal & 3 & Total P,\% $\%$ & 0.65 \\
Soybean oil & 3.5 & Available P,\% & 0.45 \\
dl-Methionine & 0.27 & Methionine + cysteine,\% & 0.9 \\
NaCl & 0.27 & Lysine,\% & 1.05 \\
Limestone & 1.33 & - & - \\
Calcium phosphate & 1.33 & - & - \\
Vitamin-mineral premix & 1 & - & - \\
\hline
\end{tabular}

a Supplied per kilogram of diet: vitamin A (retinyl acetate), 1,600 IU; cholecalciferol, $300 \mathrm{IU}$; vitamin E (DL- $\alpha$-tocopheryl acetate), $10 \mathrm{IU}$; riboflavin, $3.8 \mathrm{mg}$; pantothenic

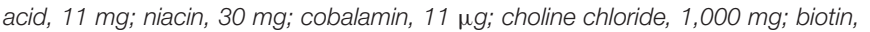
$0.16 \mathrm{mg}$; folic acid, $0.45 \mathrm{mg}$; thiamine $1.5 \mathrm{mg}$; pyridoxine $3.0 \mathrm{mg} ; \mathrm{Fe}, 68 \mathrm{mg} ; \mathrm{Zn}$, 50 mg; Mn, 60 mg; l, 0.2 mg; Cu, 8 mg; Se, 0.16 mg. 
gut microbiota and SCFAs. The cecum tissue was collected for inflammatory cytokine mRNA measurements. The serum was collected for inflammatory cytokine detection by enzyme-linked immunosorbent assay (ELISA).

\section{Viral RNA Extraction and Quantitative Real-Time Polymerase Chain Reaction}

Viral RNA was extracted from cecal content suspensions using the TRizol ${ }^{\mathrm{TM}}$ method (Invitrogen, Carlsbad, CA, United States) according to the manufacturer's instructions. The viral RNA was further reverse transcribed into cDNA. Viral RNA titers were determined using quantitative real-time polymerase chain reaction (qRT-PCR) as reported previously (Liang et al., 2019). The detection limit of the qRT-PCR was $4.6 \log 10 \mathrm{GE} / \mathrm{mL}$.

\section{DNA Extraction From the Cecal Content and $16 S$ rRNA Sequencing}

Total genomic DNA was extracted from $0.5 \mathrm{~g}$ of colonic contents using the FastDNA ${ }^{\circledR}$ SPIN Kit (MP Bio, Santa Ana, CA, United States) following the manufacturer's guidelines, with the additional glass-bead beating steps on a FastPrep 24 homogenizer (MP Biomedicals, Santa Ana, CA, United States). The final DNA concentration was quantified on a NanoDrop 2000 UV-vis spectrophotometer (Thermo Scientific, Wilmington, DE, United States). The integrity and size of genomic DNA was checked by $1.0 \%$ agarose gel electrophoresis. The DNA extracts were sequenced for $16 \mathrm{~S}$ rRNA using Illumina MiSeq (Illumina, San Diego, CA, United States), targeting the V3-V4 region with barcoded 338F (5'-ACTCCTACGGGAGGCAGCAG-3') and 806R (5'-GGACTACHVGGGTWTCTAAT-3') universal primers (Mori et al., 2013), and sequences were processed according to the standard protocols by Majorbio Bio-Pharm Technology Co. Ltd. (Shanghai, China).

\section{Determination of SCFAs}

The SCFAs in cecum were determined using gas chromatography (GC) according to a previously described method (Stewart et al., 2010). The samples were placed on a DB-WAX column $(30 \mathrm{~m}$ long $\times 0.53 \mathrm{~mm}$ diameter and $1.00 \mu \mathrm{m}$ film thickness) and were separated by using a TRACE ${ }^{\mathrm{TM}} 1300 \mathrm{GC}$ with flame ionization detector (FID). The temperature program was $100^{\circ} \mathrm{C}$ for $0.5 \mathrm{~min}$, then raised to $170^{\circ} \mathrm{C}$ at $8^{\circ} \mathrm{C} / \mathrm{min}$ and held for $1 \mathrm{~min}$, then raised to $200^{\circ} \mathrm{C}$ at $40^{\circ} \mathrm{C} / \mathrm{min}$ and held for $2 \mathrm{~min}$. Samples were run with a $30: 1$ split ratio and a $5.0 \mathrm{~mL} / \mathrm{min}$ column flow. High-purity hydrogen was used as the carrier gas. The temperatures of the injector and detector were 250 and $270^{\circ} \mathrm{C}$, respectively.

\section{Detection of Inflammatory Cytokine Expression}

The levels of expression of the inflammatory cytokines (IFN- $\gamma$, TNF- $\alpha$, and IL-10) in the cecum of SPF chicks were quantified by qRT-PCR. Cecum tissue samples were homogenized by the TISSUELYSER-24 (Jingxin, Shanghai, China). Total RNAs were extracted from the supernatant of the cecum tissue lysate by TRizol $^{\mathrm{TM}}$ Reagent Kit (Invitrogen, Carlsbad, CA, United States) and were quantified by NanoDrop 2000 spectrophotometer
(Thermo Fisher Scientific, Waltham, MA, United States), then further reverse transcribed into cDNA. The total reaction volume of $20 \mu \mathrm{L}$ contained $2.0 \mu \mathrm{L}$ of DNA, $10.0 \mu \mathrm{L}$ of SYBR Green qPCR Mix (Takara Bio Inc., Tokyo, Japan), $7 \mu \mathrm{L}$ of $\mathrm{H}_{2} \mathrm{O}$, and $0.5 \mu \mathrm{L}$ of each specific primer. Primers were synthesized according to previous reports (Emami et al., 2019) and are shown in Table 2. Reactions were performed under the following conditions: one cycle of preincubated samples at $95^{\circ} \mathrm{C}$ for $1 \mathrm{~min}$ and 39 cycles of amplification samples at $95^{\circ} \mathrm{C}$ for $5 \mathrm{~s}, 60^{\circ} \mathrm{C}$ for $30 \mathrm{~s}, 72^{\circ} \mathrm{C}$ for $30 \mathrm{~s}$. Target gene expression of each sample was normalized using glyceraldehyde 3 -phosphate dehydrogenase (GAPDH). The relative expression of the target gene for each sample was calculated using the $2^{-\Delta \Delta \mathrm{Ct}}$ method (Livak and Schmittgen, 2001).

The expression levels of the IFN- $\gamma$, TNF- $\alpha$ and IL-10 in serum were detected using ELISA Kit from Nanjing Jian cheng Biological Institute (Nanjing, China). Serum samples were obtained and measured as previously described (Huang et al., 2015). Absorbance was read at $450 \mathrm{~nm}$ using a microplate spectrophotometer. The inflammatory cytokine concentrations were calculated according to a best-fit standard curve.

\section{Data Processing and Statistical Analysis}

Raw fastq files were demultiplexed, quality-filtered by Trimmomatic, and merged by FLASH (Magoč and Salzberg, 2011). Sequences with ambiguous bases, shorter than $200 \mathrm{bp}$, and sequences with an average mass less than 25 were removed. The high-quality reads were clustered into operational taxonomic units (OTUs) with 97\% similarity cutoff using UPARSE (version 7.11) and used for further analysis of the Venn diagram, alpha diversity indices (Shannon and Simpson), and richness estimators (ACE and Chao). Beta diversity measures were based on weighted-uniFrac distance analysis and shown by principal coordinate analysis (PCoA), which was conducted to assess the relationships among the different groups. LEfSe was applied to recognize which bacterial taxa contribute to the differences between the two groups (Segata et al., 2011), and the minimal threshold of linear discriminant analysis (LDA) was set at 3.5. The difference between specific taxa was analyzed using one-way analysis of variance (ANOVA) followed by post hoc $t$-tests. Data analysis of inflammatory cytokines and SCFAs was performed using GraphPad Prism (version 6.01, La Jolla, CA, United States). A $P<0.05$ or 0.01 was considered statistically significant or highly significant, which is indicated as follows: ${ }^{*} P<0.05,{ }^{* *} P<0.01$.

\section{RESULTS}

\section{Apparent Clinical Characteristics of SPF Chicks Infected With PDCoV}

SPF chicks, intragastrically inoculated with PDCoV HNZK-02 at 4 days old, showed mild diarrhea at 5 dpi. PDCoV RNA was detected in the intestinal contents by qRT-PCR at 5 and 17 dpi while non-infected control chicks appeared normal throughout the course of the experiments. PDCoV HNZK-02 infection had 
TABLE 2 | The sequences of primers used in this study for real-time RT-PCR.

\begin{tabular}{|c|c|c|c|}
\hline Genes & Forward primer $\left(5^{\prime}\right.$ to $\left.3^{\prime}\right)$ & Reverse primer $\left(5^{\prime}\right.$ to $\left.3^{\prime}\right)$ & Amplified size (bp) \\
\hline $\mathrm{IFN}-\gamma$ & GCTCCCGATGAACGACTTGA & TGTAAGATGCTGAAGAGTTCATTCG & 63 \\
\hline TNF- $\alpha$ & CCCATCCCTGGTCCGTAAC & ATACGAAGTAAAGGCCGTCCC & 77 \\
\hline IL-10 & CGCTGTCACCGCTTCTTCA & CGTCTCCTTGATCTGCTTGATG & 63 \\
\hline GAPDH & CCTAGGATACACAGAGGACCAGGTT & GGTGGAGGAATGGCTGTCA & 64 \\
\hline
\end{tabular}

From Emami et al. (2019).

TABLE 3 | Comparison of phylotype coverage and diversity estimation of the $16 \mathrm{~S}$ rRNA gene libraries at $97 \%$ similarity from the pyrosequencing analysis.

\begin{tabular}{|c|c|c|c|c|c|c|c|}
\hline \multirow[t]{2}{*}{ Group } & \multirow[t]{2}{*}{ No. of reads } & \multirow[t]{2}{*}{ No. of OTUs } & \multirow[t]{2}{*}{ Coverage (\%) } & \multicolumn{2}{|c|}{ Richness estimator } & \multicolumn{2}{|c|}{ Diversity index } \\
\hline & & & & $\begin{array}{c}\text { ACE } \\
(\text { mean } \pm \text { SE) }\end{array}$ & $\begin{array}{c}\text { Chao } \\
\text { (mean } \pm \text { SE) }\end{array}$ & $\begin{array}{c}\text { Shannon } \\
(\text { mean } \pm \text { SE) }\end{array}$ & $\begin{array}{c}\text { Simpson } \\
(\text { mean } \pm \text { SE })\end{array}$ \\
\hline Mock_5d & 179,564 & 277 & 99.95 & $226.37 \pm 2.67^{c}$ & $231.41 \pm 5.95^{b}$ & $3.58 \pm 0.16$ & $0.057 \pm 0.13$ \\
\hline PDCoV HNZK_02_5d & 160,137 & 325 & 99.93 & $282.33 \pm 0.51^{b}$ & $293.29 \pm 2.87^{a}$ & $3.53 \pm 0.11$ & $0.072 \pm 0.15$ \\
\hline Mock_17d & 168,425 & 341 & 99.95 & $303.29 \pm 13.75^{a, b}$ & $302.96 \pm 16.46^{a}$ & $3.82 \pm 0.06$ & $0.054 \pm 0.09$ \\
\hline PDCoV HNZK_02_17d & 155,610 & 351 & 99.93 & $311.57 \pm 7.58^{a}$ & $318.37 \pm 9.47^{a}$ & $3.62 \pm 0.06$ & $0.077 \pm 0.08$ \\
\hline
\end{tabular}

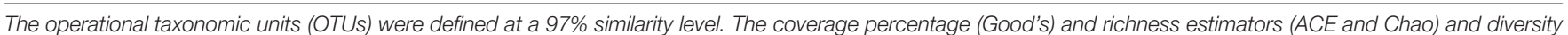

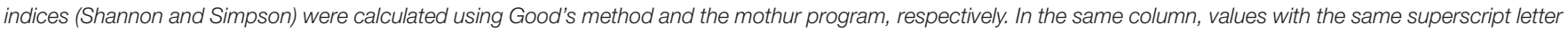
are not significantly different $(P>0.05)$; those with different superscript letters differ significantly $(P<0.05)$.

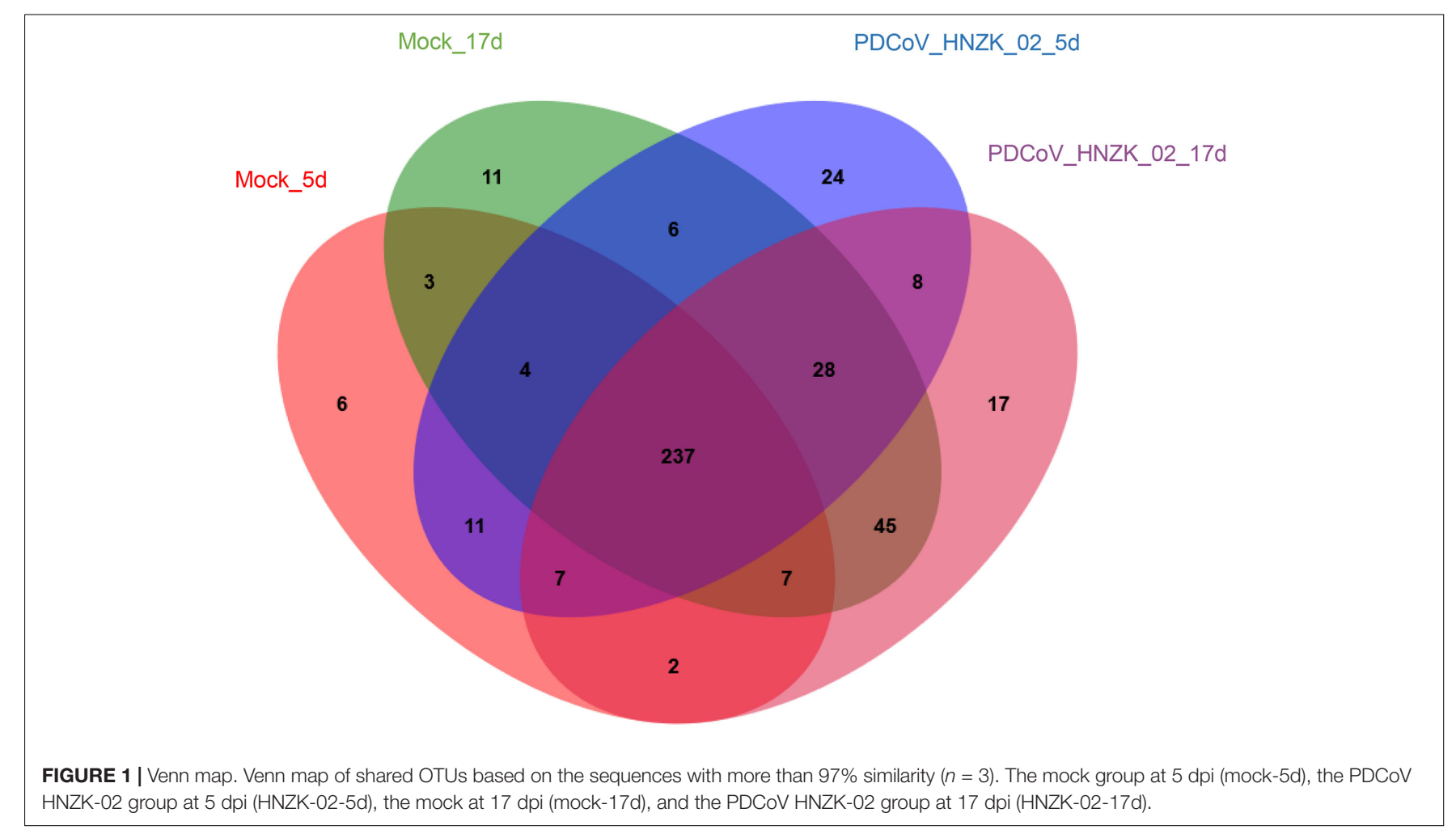

no effect on feed consumption compared to controls during the whole experimental period.

\section{Characteristics of Pyrosequencing Results}

A total of 12 cecal concent samples, the control chicks $(n=3)$, and PDCoV HNZK-02 groups $(n=3)$ at 5 and 17 dpi, respectively, were evaluated using $16 \mathrm{~S}$ rRNA gene sequencing. A total of 663,736 sequences with a median read length of 429 bp (range from 274 to $494 \mathrm{bp}$ ) were collected. The total number of unique sequences from the four groups was 416 . The sequence and species-level OTUs of each group are shown in Table 3. The Venn diagram showed that 237 OTUs of the total gut microbial richness (416) were shared among all the sequenced samples. Two hundred and fifty-nine OTUs were shared between the samples 
of the mock and PDCoVHNZK-02 groups with a total of 343 at 5 dpi. Three hundred and seventeen OTUs of the total gut microbial richness (375) were shared between the samples of mock and PDCoV HNZK-02 groups at 17 dpi (Figure 1).

\section{Alpha and Beta Diversity Analyses}

Alpha diversity estimators of community are shown in Table 3. Gut microbial richness, according to the Ace and Chao indexes, was significantly increased in the PDCoV HNZK-02 group when compared with that of the mock group at $5 \mathrm{dpi}(P<0.05)$, while there was no significant difference between the two groups at $17 \mathrm{dpi}(P>0.05)$. In Shannon and Simpson alpha diversity indexes, the differences between the PDCoV HNZK-02 and the mock groups at 5 and $17 \mathrm{dpi}$ did not reach statistical significance $(P>0.05)$.

Beta diversity analysis showed that there were some similarities in the microbial composition between the mock and the PDCoV HNZK-02 groups at 5 dpi. However, the microbial composition from chicks with $\mathrm{PDCoV}$ inoculation and the controls could be divided into two different clusters at $17 \mathrm{dpi}$, indicating that the cecal microbial community structure and composition in the control group were significantly different from those in the PDCoV HNZK-02 group at $17 \mathrm{dpi}$ (Figure 2).

\section{Difference in Specific Taxa Between PDCoV HNZK-02-Infected Chicks and Controls}

To further investigate whether PDCoV HNZK-02 infected chicks experienced any significant alteration in gut microbiota, we analyzed the relative abundance of microbiota at the phylum, family, and genus levels. At $5 \mathrm{dpi}$, genus level analysis showed the taxonomic abundance was altered in the PDCoV HNZK02 group, which was characterized by lower abundance of Eisenbergiella and Anaerotruncus $(P<0.05)$. There were no significant differences at the levels of phylum and family between the mock and the PDCoV HNZk-02 groups (Figure 3A). At $17 \mathrm{dpi}$, the ratio of Firmicutes to Bacteroidetes in the PDCoV HNZk-02 group was lower than that of the mock group. At the family level, the proportion of Rikenellaceac in the chicks inoculated with PDCoV HNZK-02 significantly increased $(P<0.05)$. At the genus level, Alistipes and the unclassified f-Ruminococcaceae were significantly increased $(P<0.05)$, whereas the proportion of RuminococcaceaeUGG-014 was significantly decreased in the PDCoV HNZK-02 group when compared to that of the mock group at $17 \mathrm{dpi}$ $(P<0.01)$ (Figure 3B).

The characteristics of bacterial taxonomic abundant in the collected samples were further analyzed using LEfSe algorithm (Figure 4). LEfSe analysis revealed that there existed main 8 and 33 discriminative features from phylum to genus (LDA score $>3$ ) between the mock and the PDCoV HNZK-02-inoculated chicks at 5 and 17 dpi (Figures 4A,B), respectively. More specifically, Eisenbergiella and Anaerotruncus were the most differential microbiota in the mock at 5 dpi. Moraxellaceae, Acinetobacter, Pseudomonadates, Eubacterium, and norank-f Lachnospiraceae in the PDCoV HNZK-02 group increased when compared to that in the mock group. At $17 \mathrm{dpi}$, we identified 10 microbial biomarkers from the PDCoV HNZK-02 group, including Rikenellaceae, Bacteroidia, Bacteroidetes, Bacteroidales, Alistipes, unclassified-f-Ruminocococcaceae, Sphingomonadaceae, Novosphingobium, and Lachnoclostridium,

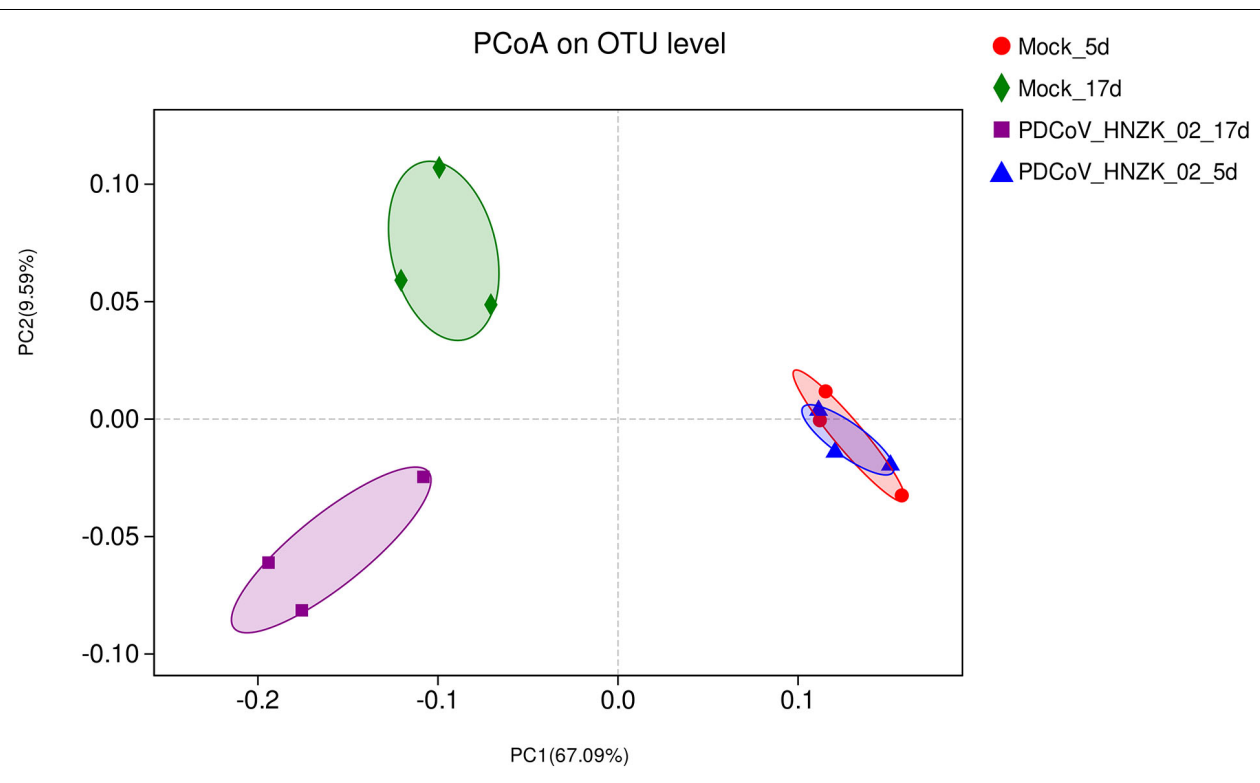

FIGURE 2 | Beta diversity measures in cecal microbiota of chickens $(n=3)$. The scatterplot of the principal coordinate analysis (PCoA) scores shows four different clusters (weighted-uniFrac distance), the blue represents the PDCoV HNZK-02 group at 5 dpi (HNZK-02-5d), the red represents the mock group at 5 dpi (mock-5d), the purple represents the PDCoV HNZK-02 group at 17 dpi (HNZK-02-17d), and the green represents the mock group at 17 dpi (mock-17d). 


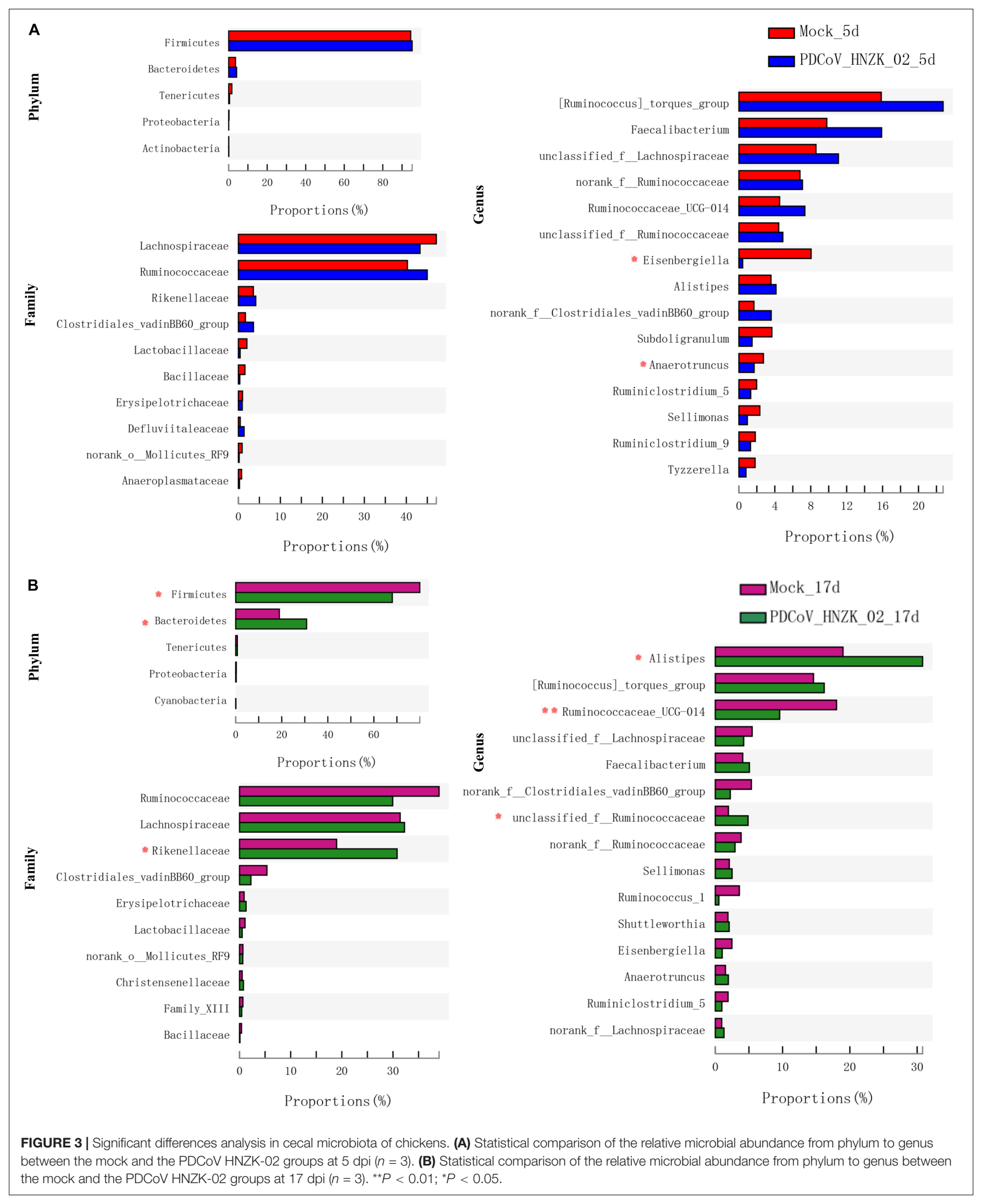




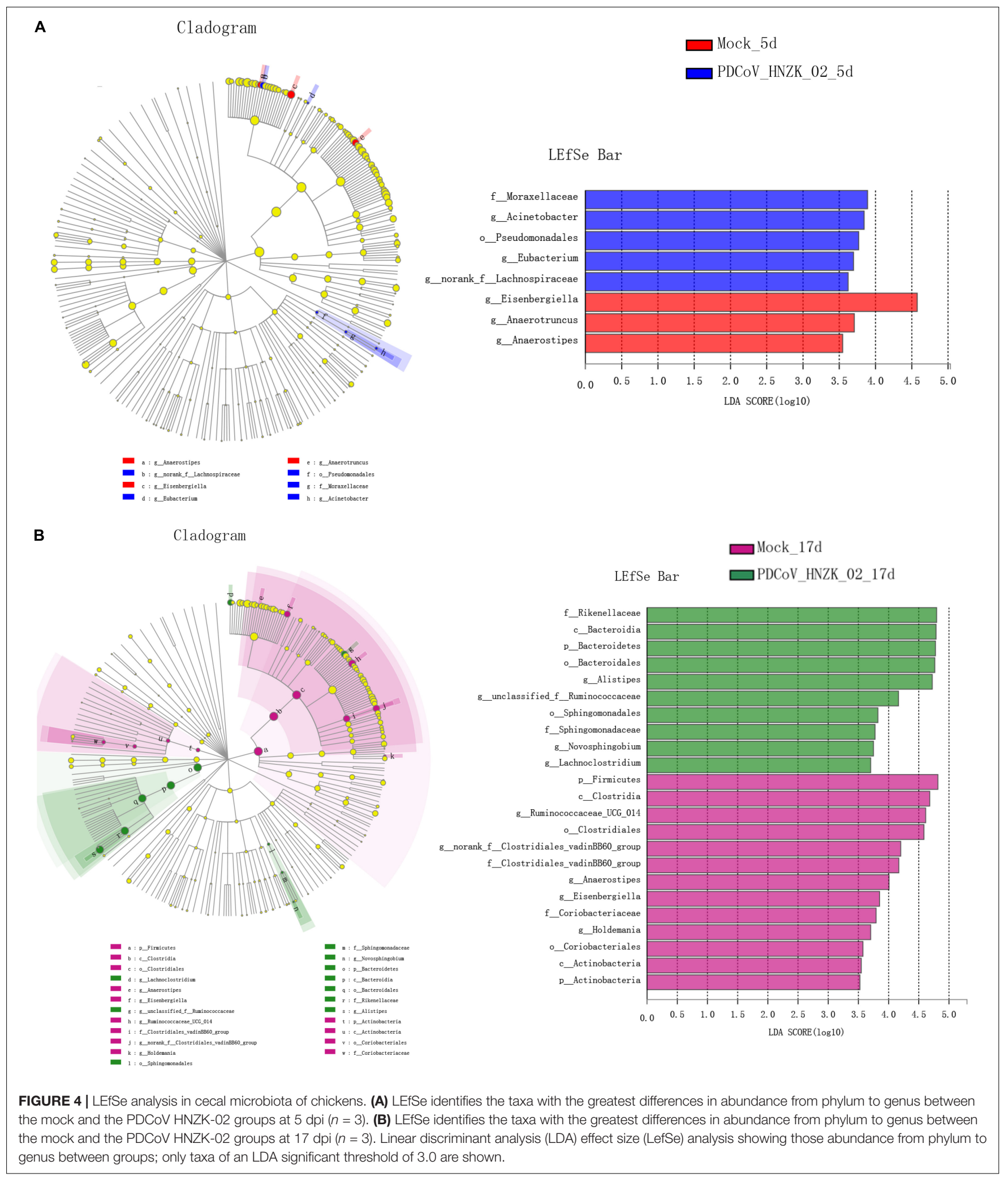

which could be distinguished from the mock group. 13 clades were more abundant in the mock group than that in the PDCoV HNZK-02 group, including Firmicutes,
Clostridia, Ruminocococaceae-UCG-014, norank-f-Clostridiales-vadinBB60-group, Clostridia, ClostridialesvadinBB60-group, Anaerostipe, Eisenbergiella, Coriobacteriaceae, 


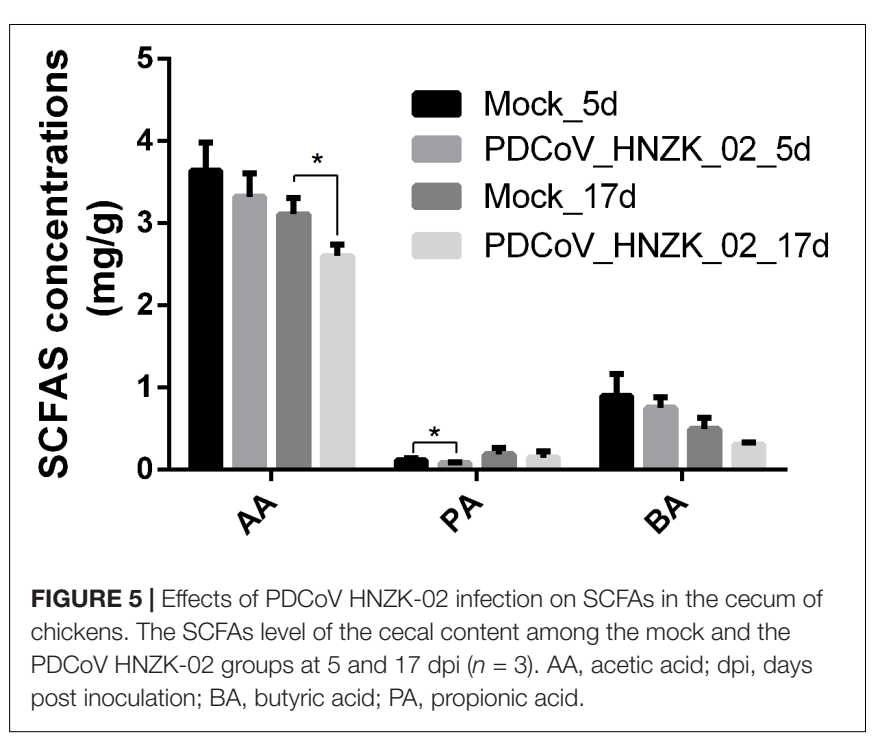

Holdemania, Coriobacteriales, Actinobacteria, and Actinobacteria. There were bigger differences on microbiota compositions between the early and later stages of PDCoV infection (at 5 and $17 \mathrm{dpi}$, respectively).

\section{SCFA Concentration in Cecal Content of Chicks Inoculated With PDCoV}

Compared to the control, there was a decreasing trend in the amounts of acetic acid (AA), propionic acid (PA), and butyric acid (BA) in the PDCoV HNZK-02 group at 5 and 17 dpi. Furthermore, PA and AA levels in the PDCoV HNZK02 group were greatly decreased at 5 and $17 \mathrm{dpi}$, respectively $(P<0.05)$ (Figure 5).

\section{PDCoV HNZK-02 Infection Promotes the Expression of Inflammatory Cytokines in Chicks}

As shown in Figure 6, the expression of IFN- $\gamma(P<0.01)$, TNF$\alpha(P<0.05)$ and IL-10 $(P<0.01)$ was significantly upregulated at 5 dpi compared with that in the mock chicks (Figures 6AC). Furthermore, IFN- $\gamma$ expression was significantly upregulated (Figure 6A) at $17 \mathrm{dpi}(P<0.01)$.

To evaluate if PDCoV infection could affect IFN- $\gamma$, TFN- $\alpha$, and IL-10 secretion in serum, the concentrations of IFN- $\gamma$, TFN$\alpha$, and IL-10 in serum were tested with ELISA kits. Our results showed that the changes of the inflammatory cytokines in serum were similar with those in the cecum tissue. The levels of IFN$\gamma$ and TNF- $\alpha$ were approximately two and three times as high as in the PDCoV HNZK-02 group when compared to that of the mock group at $5 \mathrm{dpi}(P<0.01)$. The level of IL-10 in the $\mathrm{PDCoV}$ infection group was also increased at $5 \mathrm{dpi}(P<0.05)$. The serum levels of IFN- $\gamma$ in the PDCoV-infected chicks were higher than in the mock group at $17 \mathrm{dpi}(P<0.01)$, and no significant differences were observed for the secretion of TNF$\alpha$ and IL-10 between the mock and the PDCoV HNZK-02 groups at $17 \mathrm{dpi}$ (Figures 7A-C).

\section{Relationship Between Microbial Signatures and the Levels of SCFAs or the Expression of Inflammatory Cytokines in Chicks Infected With PDCoV}

Spearman's correlation analysis was conducted between the top 15 bacterial genera and the environmental factors (SCFAs, proinflammatory cytokines), and was directly reflected by a heatmap (Figure 8). The threshold $|R|>0.8$ was considered as having a correlation. The results indicated that Eisenbergiella was negatively correlated with TNF$\alpha(P<0.05)$ and IFN- $\gamma(P<0.01)$ expression levels, and was positively correlated with PA $(P<0.01)$ at 5 dpi (Figure 8A). The Ruminiclostridium_9 also showed a positively correlation with the IL-10 expression $(P<0.05)$, while Norank_f_ Ruminococcaceae was negatively correlated with BA $(P<0.001)$. At 17 dpi (Figure 8B), Alistipes and unclassified_f_ Ruminococcaceae showed an extremely significant positive correlation with IFN- $\gamma$ secretion $(P<0.05)$, but Eisenbergiella and Norank_f_Clostridiales_vadinbb60_group were negatively correlated with it $(P<0.05)$. The level of PA was associated with the increase in the abundance of Ruminiclostridium_5 $(P<0.05)$. Ruminococcus and Eisenbergiella were positively correlated with AA $(P<0.05)$, while Alistipes showed a negative relationship with it $(P<0.05)$. Eisenbergiella, unclassified_f_Lachnospiraceae, Ruminococcaceae_UCG-014, Norank_f_Clostridiales_vadinbb60_group, and Ruminiclostr idium_ 5 were positively correlated with BA.

\section{DISCUSSION}

Gut microflora play an important role in shaping immunity against viral diseases in host. Disruption of microbial homeostasis is associated with a variety of pathological states, which helps the establishment of acute viral infections in chickens (Abaidullah et al., 2019). In this study, the infection model of PDCoV was developed on SPF chicks. Our results demonstrated similar clinical diseases, and the dynamics of the virus shedding in cecum were observed in all SPF chicks inoculated with the PDCoV HNZK-02 strains when compared with our previous experiment (Liang et al., 2019). The influence of PDCoV infection on cecum microbiota of SPF chicken was evaluated, PDCoV HNZK-02 infection significantly altered the gut microbiota composition and decreased SCFAs products in chicks' cecum. Simultaneously, the expression levels of inflammatory cytokines (IFN- $\gamma$, IL-10, and TNF- $\alpha$ ) in serum and cecum tissue were significantly increased, resulting in the inflammatory response in the chicken. The analysis showed a significant correlation between bacterial genera and SCFAs or inflammatory cytokines.

The diversity of indigenous intestinal microbiota is one of the key factors in resisting the colonization of invading pathogens (Keesing et al., 2010). Several studies have reported that the diarrhea-relating coronaviruses could influence the intestinal microbiota diversity in pigs (Liu et al., 2015; Song et al., 2017; 

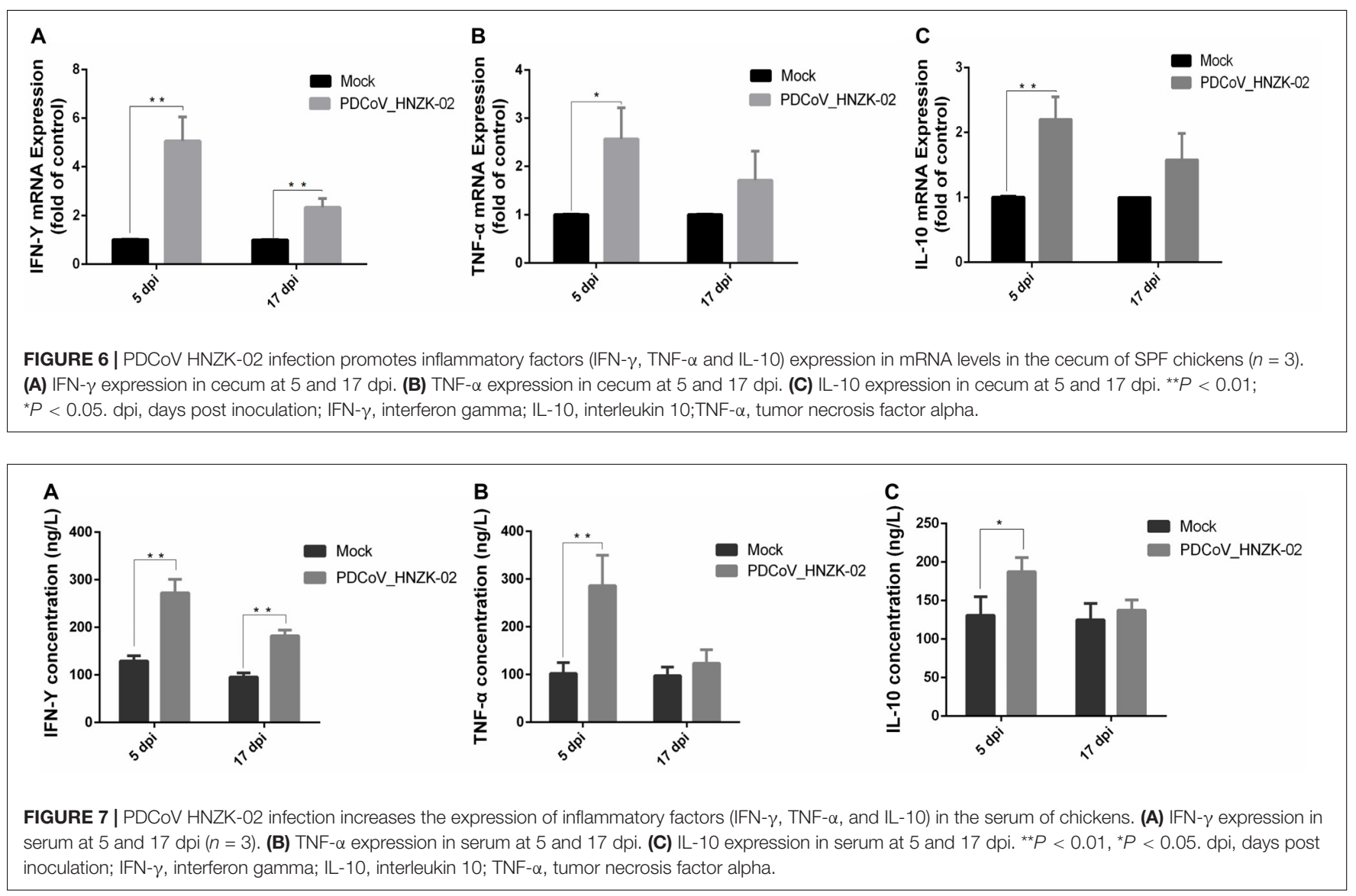

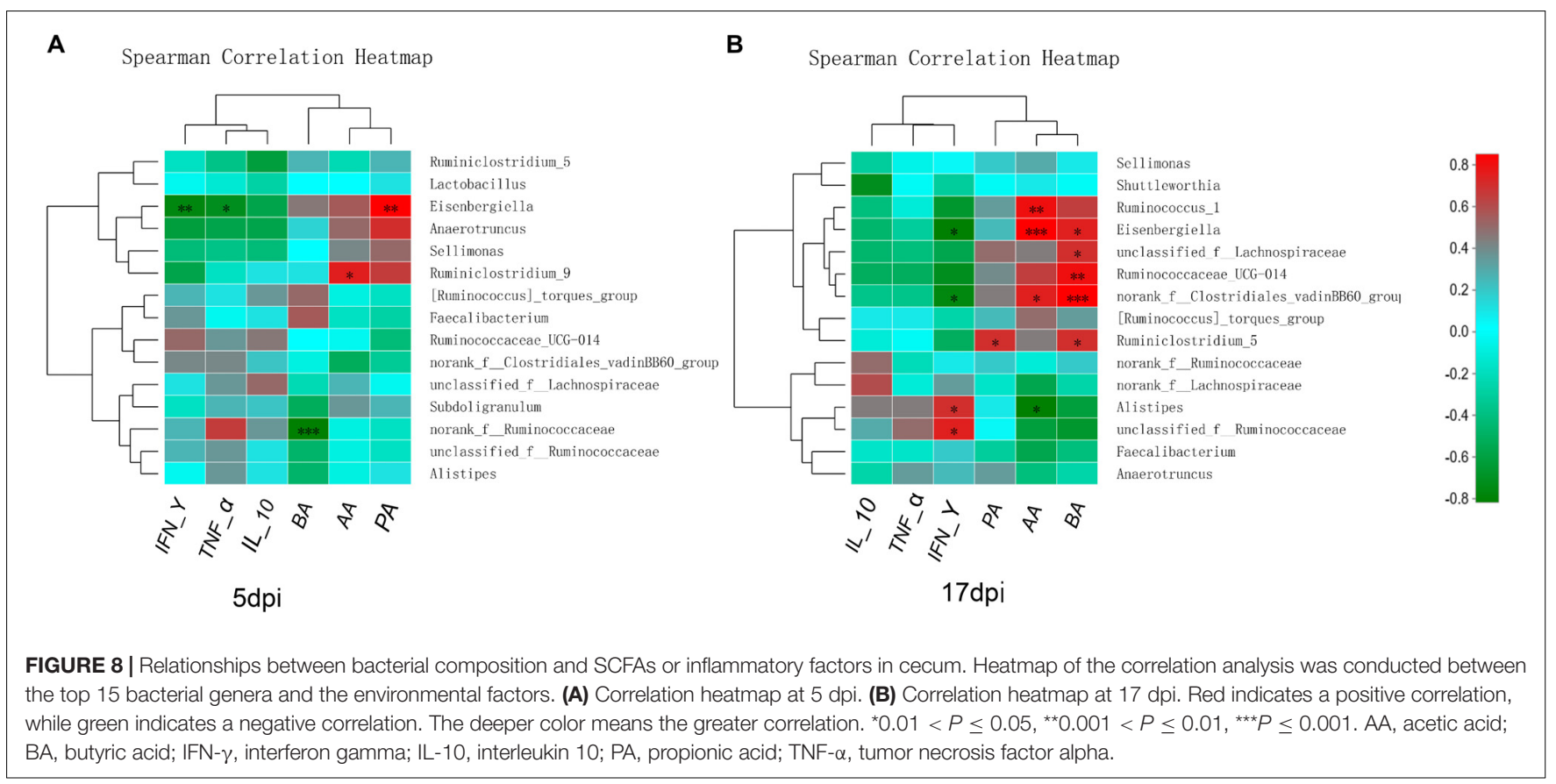

Huang et al., 2018; Tan et al., 2019). Indeed, many viral agents have been shown to alter the intestinal microbiota in chickens, such as AIV (Yitbarek et al., 2018), Marek's disease virus (MDV) (Lillehoj and Trout, 1996), NDV (Li, 2007), and IBDV (Li L. et al., 2018). In PDCoV-infected chicks, the species richness indices were increased at $5 \mathrm{dpi}$, and 
there were no significant changes in the overall $\alpha$-diversity of cecal microbiota at $17 \mathrm{dpi}$. It is possible that the reduced immune response may account for the change of microbial diversity. From the PCoA results, microbiota structure in the PDCoV infected group was clearly distinguished from that of the mock group at $17 \mathrm{dpi}$, which also reflected SPF chicks' resistance to $\mathrm{PDCoV}$.

From microbial community profiling, the difference between the groups was analyzed at the phylum, family, and genus level. Eisenbergiella and Anaerotruncus genera were significantly decreased in the PDCoV HNZK-02 at $5 \mathrm{dpi}$, indicating that PDCoV HNZK-02 infection had a marked influence on chicks' cecal microbiota. According to previous studies, the decrease of the ratio of Firmicutes-to-Bacteroidetes was observed in mice with diabetes (Wen et al., 2008) and in some patients with Crohn's disease and ulcerative colitis (Frank et al., 2007). In our results, a decreased ratio of Firmicutes-to-Bacteroidetes occurred in the PDCoV HNZK-02 group at $17 \mathrm{dpi}$. More remarkably, the Alistipes, considered an opportunistic pathogen (Pandit et al., 2018; Zhang et al., 2018), was significantly increased in the PDCoV HNZK-02 group at $17 \mathrm{dpi}$. We used linear discriminant analysis (LDA) of effect size (LEfSe) to confirm the taxa that most likely explains the differences between the PDCoV HNZK-02 and mock groups. These findings provide compelling evidence that PDCoV HNZK02 induced microbiota imbalance and showed that recovery was difficult in a short time period after PDCoV HNZK02 infection.

Production of SCFAs, as the end products of protein and carbohydrate fermentation (Cummings and Macfarlane, 1991), can easily be affected by the status of the gut microbiota (Lloyd-Price et al., 2019). Studies have shown that Lactobacillus salivarius and $L$. agilis could increase propionate and butyrate contents in cecum of chicks (Meimandipour et al., 2010). The changes in butyrate and propionate can influence intestinal physiology and immune function, while acetate can alter lipid metabolism (Macfarlane and Macfarlane, 2011). Our data showed that the changes of gut microbiota composition contributed to the decreased SCFAs, which reduced this protective effect of SCFA in the cecum. However, the interaction of gut microbiota with SCFAs to respond to PDCoV infection needs further studies.

Inflammatory immune responses in the gut can alter the gut luminal environment in a way that favors dysbiosis (Winter et al., 2013). Chicks with dysbiosis are more prone to acute viral infection (Abaidullah et al., 2019), which may in turn further affect the pathogen infectivity. Eisenbergiella is strongly correlated with increased levels of TNF- $\alpha$ and IFN- $\gamma$, which help in modulating the functional activities of the cells of the immune system (Liu et al., 2010), and to block virus replication and prevent clinical disease (Bradley, 2008; Praveena et al., 2010). This may be the reason that Eisenbergiella was decreased in the PDCoV HNZK-02 group at 5 dpi. Moreover, the observation is similar to the increased proinflammatory (TNF- $\alpha$ ) cytokine responses of 10-day-old Gn pigs to acute PDCoV infection (Jung et al., 2018). Alistipes was strong positively correlated with IFN- $\gamma$ levels, and the numbers of Ruminococcaceae_UCG-014 were strong negatively correlated with IFN- $\gamma$ levels, indicating that these bacteria can be tolerated, with IFN- $\gamma$ responses benefiting for those genera of bacteria. The anti-inflammation activity could be explained in the study regarding to the administration of Norank_f_Lachnospiraceae in gut, and was related to the IL-10 cytokine (Olszak et al., 2014). The results showed the change of microbiota in the cecum was related to intestinal inflammation (directly or indirectly, positively or negatively).

\section{CONCLUSION}

In conclusion, the present study revealed that obvious changes were found in the microbial community of chicks infected with PDCoV HNZK-02. PDCoV-inoculated chicks showed a decrease in Eisenbergiella and Anaerotruncus populations at 5 dpi and an increase in pathogenetic Alistipes at $17 \mathrm{dpi}$ with a reduction in SCFAs production. The changes in the microbial community may be related to changes in inflammatory cytokine expression. These results provide new insights into the pathophysiology of chicks infected with PDCoV.

\section{DATA AVAILABILITY STATEMENT}

The obtained raw sequencing reads have been added to the NCBI SRA page (Accession Number: PRJNA558384). Other data generated during the study are included in this article.

\section{ETHICS STATEMENT}

The experimental procedures were approved by the Laboratory Animals Ethics Committee at Hennan Qixiang Biological Technology Co. Ltd. (HNQX-2018-06), and all husbandry practices and euthanasia were performed with full consideration of animal welfare.

\section{AUTHOR CONTRIBUTIONS}

HL and HZ performed the experiments. HL, FZ, and SW performed statistical analyses and wrote the manuscript. Z-xW and Z-yW designed the study and revised the manuscript. All authors read and approved the final manuscript.

\section{FUNDING}

This work was supported by the National Key R\&D Program (2018YFD0500102) and the National Natural Science Foundation of China (U1704231).

\section{ACKNOWLEDGMENTS}

The authors thank Dr. Hui $\mathrm{Hu}$ from Henan Agricultural University for designing the study and revising the manuscript. 


\section{REFERENCES}

Abaidullah, M., Peng, S., Kamran, M., Song, X., and Yin, Z. (2019). Current findings on gut microbiota mediated immune modulation against viral diseases in chicken. Viruses 11:681. doi: 10.3390/v11080681

Abt, M. C., Osborne, L. C., Monticelli, L. A., Doering, T. A., Alenghat, T., Sonnenberg, G. F., et al. (2012). Commensal bacteria calibrate the activation threshold of innate antiviral immunity. Immunity 37, 158-170. doi: 10.1016/j. immuni.2012.04.011

Balamurugan, V., and Kataria, J. (2006). Economically important non-oncogenic immunosuppressive viral diseases of chicken-current status. Vet. Res. Commun. 30, 541-566.

Boley, P. A., Alhamo, M. A., Lossie, G., Yadav, K. K., Vasquez-Lee, M., Saif, L. J., et al. (2020). Porcine deltacoronavirus infection and transmission in poultry, United States. Emerg. Infect. Dis. 26, 255-265. doi: 10.3201/eid2602.190346

Bradley, J. (2008). TNF-mediated inflammatory disease. J. Pathol. 214, 149-160.

Budden, K. F., Gellatly, S. L., Wood, D. L., Cooper, M. A., Morrison, M., Hugenholtz, P., et al. (2017). Emerging pathogenic links between microbiota and the gut-lung axis. Nat. Rev. Microbiol. 15, 55-63.

Chen, C.-J., Wu, G.-H., Kuo, R.-L., and Shih, S.-R. (2017). Role of the intestinal microbiota in the immunomodulation of influenza virus infection. Microbes Infect. 19, 570-579. doi: 10.1016/j.micinf.2017.09.002

Chen, Q., Li, G., Stasko, J., Thomas, J. T., Stensland, W. R., Pillatzki, A. E., et al. (2014). Isolation and characterization of porcine epidemic diarrhea viruses associated with the 2013 disease outbreak among swine in the United States. J. Clin. Microbiol. 52, 234-243.

Cummings, J., and Macfarlane, G. (1991). The control and consequences of bacterial fermentation in the human colon. J. Appl. Bacteriol. 70, 443-459.

Deriu, E., Boxx, G. M., He, X., Pan, C., Benavidez, S. D., Cen, L., et al. (2016). Influenza virus affects intestinal microbiota and secondary salmonella infection in the gut through type I interferons. PLoS Pathog. 12:e1005572. doi: 10.1371/ journal.ppat.1005572

Donohoe, D. R., Holley, D., Collins, L. B., Montgomery, S. A., Whitmore, A. C., Hillhouse, A., et al. (2014). A gnotobiotic mouse model demonstrates that dietary fiber protects against colorectal tumorigenesis in a microbiota-and butyrate-dependent manner. Cancer Discov. 4, 1387-1397. doi: 10.1158/21598290.CD-14-0501

Emami, N. K., Calik, A., White, M. B., Young, M., and Dalloul, R. A. (2019), Necrotic enteritis in broiler chickens: the role of tight junctions and mucosal immune responses in alleviating the effect of the disease. Microorganisms 7:231. doi: 10.3390/microorganisms7080231

Frank, D. N., Amand, A. L. S., Feldman, R. A., Boedeker, E. C., Harpaz, N., and Pace, N. R. (2007). Molecular-phylogenetic characterization of microbial community imbalances in human inflammatory bowel diseases. Proc. Natl. Acad. Sci. U.S.A. 104, 13780-13785.

Hu, H., Jung, K., Vlasova, A., and Saif, L. (2016). Experimental infection of gnotobiotic pigs with the cell-culture-adapted porcine deltacoronavirus strain OH-FD22. Arch. Virol. 161, 1-14.

Huang, J., Zhang, Z., Li, M., Song, X., Yan, R., Xu, L., et al. (2015). Eimeria maxima microneme protein 2 delivered as DNA vaccine and recombinant protein induces immunity against experimental homogenous challenge. Parasitol. Int. 64, 408-416. doi: 10.1016/j.parint.2015.06.002

Huang, M.-Z., Wang, S.-Y., Wang, H., Cui, D.-A., Yang, Y.-J., Liu, X.-W., et al. (2018). Differences in the intestinal microbiota between uninfected piglets and piglets infected with porcine epidemic diarrhea virus. PLoS One 13:e0192992. doi: 10.1371/journal.pone.0192992

Jung, K., Hu, H., Eyerly, B., Lu, Z., Chepngeno, J., and Saif, L. J. (2015). Pathogenicity of 2 porcine deltacoronavirus strains in gnotobiotic pigs. Emerg. Infect. Dis. 21, 650-654. doi: 10.3201/eid2104.141859

Jung, K., Hu, H., and Saif, L. J. (2017). Calves are susceptible to infection with the newly emerged porcine deltacoronavirus, but not with the swine enteric alphacoronavirus, porcine epidemic diarrhea virus. Arch. Virol. 162, 2357-2362. doi: 10.1007/s00705-017-3351-z

Jung, K., Miyazaki, A., Hu, H., and Saif, L. J. (2018). Susceptibility of porcine IPEC-J2 intestinal epithelial cells to infection with porcine deltacoronavirus $(\mathrm{PDCoV})$ and serum cytokine responses of gnotobiotic pigs to acute infection with IPEC-J2 cell culture-passaged PDCoV. Vet. Microbiol. 221, 49-58. doi: 10.1016/j.vetmic.2018.05.019
Keesing, F., Belden, L. K., Daszak, P., Dobson, A., Harvell, C. D., Holt, R. D., et al. (2010). Impacts of biodiversity on the emergence and transmission of infectious diseases. Nature 468, 647-652. doi: 10.1038/nature0 9575

Li, B., Zheng, L., Li, H., Ding, Q., Wang, Y., and Wei, Z. (2019). Porcine deltacoronavirus causes diarrhea in various ages of field-infected pigs in China. Biosci. Rep. 39:BSR20190676. doi: 10.1042/BSR20190676

Li, H., Liu, X., Chen, F., Zuo, K., Wu, C., Yan, Y., et al. (2018). Avian influenza virus subtype H9N2 affects intestinal microbiota, barrier structure injury, and inflammatory intestinal disease in the chicken ileum. Viruses 10:270. doi: 10. 3390/v10050270

Li, L., Kubasova, T., Rychlik, I., Hoerr, F. J., and Rautenschlein, S. (2018). Infectious bursal disease virus infection leads to changes in the gut associated-lymphoid tissue and the microbiota composition. PLoS One 13:e0192066. doi: 10.1371/ journal.pone.0192066

Li, Y. (2007). ERIC-PCR Based Fingerprint to Analyze the Structural Features of Intestinal Microbial Communities of Chicks Infected with Salmonella gallinarum, Newcastle Disease Virus and the Healthy Chicks. Ph. D. thesis, Sichuan Agricultural University, Sichuan.

Liang, Q., Zhang, H., Li, B., Ding, Q., Wang, Y., Gao, W., et al. (2019). Susceptibility of chickens to porcine deltacoronavirus infection. Viruses 11:573.

Lillehoj, H. S., and Trout, J. M. (1996). Avian gut-associated lymphoid tissues and intestinal immune responses to Eimeria parasites. Clin. Microbiol. Rev. 9, 349-360.

Liu, H., Zhang, M., Han, H., Yuan, J., and Li, Z. (2010). Comparison of the expression of cytokine genes in the bursal tissues of the chickens following challenge with infectious bursal disease viruses of varying virulence. Virol. J. 7:364. doi: 10.1186/1743-422X-7-364

Liu, S., Zhao, L., Zhai, Z., Zhao, W., Ding, J., Dai, R., et al. (2015). Porcine epidemic diarrhea virus infection induced the unbalance of gut microbiota in piglets. Curr. Microbiol. 71, 643-649. doi: 10.1007/s00284-0150895-6

Livak, K. J., and Schmittgen, T. D. (2001). Analysis of relative gene expression data using real-time quantitative PCR and the 2- $\Delta \Delta$ CT method. Methods 25, 402-408.

Lloyd-Price, J., Arze, C., Ananthakrishnan, A. N., Schirmer, M., Avila-Pacheco, J., Poon, T. W., et al. (2019). Multi-omics of the gut microbial ecosystem in inflammatory bowel diseases. Nature 569, 655-662. doi: 10.1038/s41586-0191237-9

Louis, P., Hold, G. L., and Flint, H. J. (2014). The gut microbiota, bacterial metabolites and colorectal cancer. Nat. Rev. Microbiol. 12, 661-672. doi: 10. 1038/nrmicro3344

Macfarlane, G. T., and Macfarlane, S. (2011). Fermentation in the human large intestine: its physiologic consequences and the potential contribution of prebiotics. J. Clin. Gastroenterol. 45, S120-S127. doi: 10.1097/MCG. 0b013e31822fecfe

Magoč, T., and Salzberg, S. L. (2011). FLASH: fast length adjustment of short reads to improve genome assemblies. Bioinformatics 27, 2957-2963. doi: 10.1093/ bioinformatics/btr507

Meimandipour, A., Hair-Bejo, M., Shuhaimi, M., Azhar, K., Soleimani, A., Rasti, B., et al. (2010). Gastrointestinal tract morphological alteration by unpleasant physical treatment and modulating role of Lactobacillus in broilers. Br. Poult. Sci. 51, 52-59. doi: 10.1080/00071660903394455

Mori, H., Maruyama, F., Kato, H., Toyoda, A., Dozono, A., Ohtsubo, Y., et al. (2013). Design and experimental application of a novel non-degenerate universal primer set that amplifies prokaryotic 16S rRNA genes with a low possibility to amplify eukaryotic rRNA genes. DNA Res. 21, 217-227. doi: 10. 1093/dnares/dst052

National Research Council [NRC] (1994). Nutritional Requirements of Poultry. 9th Rev. Washington, DC: National Academy Press.

Olszak, T., Neves, J. F., Dowds, C. M., Baker, K., Glickman, J., Davidson, N. O., et al. (2014). Protective mucosal immunity mediated by epithelial CD1d and IL-10. Nature 509, 497-502. doi: 10.1038/nature13150

Pandit, R. J., Hinsu, A. T., Patel, N. V., Koringa, P. G., Jakhesara, S. J., Thakkar, J. R., et al. (2018). Microbial diversity and community composition of caecal microbiota in commercial and indigenous Indian chickens determined using 16s rDNA amplicon sequencing. Microbiome 6:115. doi: 10.1186/s40168-0180501-9 
Praveena, P. E., Periasamy, S., Kumar, A., and Singh, N. (2010). Cytokine profiles, apoptosis and pathology of experimental Pasteurella multocida serotype A1 infection in mice. Res. Vet. Sci. 89, 332-339. doi: 10.1016/j.rvsc.2010.04.012

Segata, N., Izard, J., Waldron, L., Gevers, D., Miropolsky, L., Garrett, W. S., et al. (2011). Metagenomic biomarker discovery and explanation. Genome Biol. 12:R60. doi: 10.1186/gb-2011-12-6-r60

Song, D., Peng, Q., Chen, Y., Zhou, X., Zhang, F., Li, A., et al. (2017). Altered gut microbiota profiles in sows and neonatal piglets associated with porcine epidemic diarrhea virus infection. Sci. Rep. 7:17439. doi: 10.1038/s41598-01717830-z

Stewart, M. L., Nikhanj, S. D., Timm, D. A., Thomas, W., and Slavin, J. L. (2010). Evaluation of the effect of four fibers on laxation, gastrointestinal tolerance and serum markers in healthy humans. Ann. Nutr. Metab. 56, 91-98. doi: $10.1159 / 000275962$

Tan, Z., Dong, W., Ding, Y., Ding, X., Zhang, Q., and Jiang, L. (2019). Changes in cecal microbiota community of suckling piglets infected with porcine epidemic diarrhea virus. PLoS One 14:e0219868. doi: 10.1371/journal.pone.0219868

Wang, L., Byrum, B., and Zhang, Y. (2014). Detection and genetic characterization of deltacoronavirus in pigs, Ohio, USA, 2014. Emerg. Infect. Dis. 20, 1227-1230. doi: 10.3201/eid2007.140296

Wen, L., Ley, R. E., Volchkov, P. Y., Stranges, P. B., Avanesyan, L., Stonebraker, A. C., et al. (2008). Innate immunity and intestinal microbiota in the development of Type 1 diabetes. Nature 455, 1109-1113. doi: 10.1038/ nature 07336

Winter, S. E., Lopez, C. A., and Bäumler, A. J. (2013). The dynamics of gut-associated microbial communities during inflammation. EMBO Rep. 14, 319-327. doi: 10.1038/embor.2013.27
Woo, P. C., Lau, S. K., Lam, C. S., Lau, C. C., Tsang, A. K., Lau, J. H., et al. (2012). Discovery of seven novel Mammalian and avian coronaviruses in the genus deltacoronavirus supports bat coronaviruses as the gene source of alphacoronavirus and betacoronavirus and avian coronaviruses as the gene source of gammacoronavirus and deltacoronavirus. J. Virol. 86, 3995-4008. doi: 10.1128/JVI.06540-11

Yitbarek, A., Taha-Abdelaziz, K., Hodgins, D. C., Read, L., Nagy, É., Weese, J. S., et al. (2018). Gut microbiota-mediated protection against influenza virus subtype H9N2 in chickens is associated with modulation of the innate responses. Sci. Rep. 8:16367. doi: 10.1038/s41598-018-34065-8

Zhang, J. (2016). Porcine deltacoronavirus: overview of infection dynamics, diagnostic methods, prevalence and genetic evolution. Virus Res. 226, 71-84. doi: 10.1016/j.virusres.2016.05.028

Zhang, W., Jiao, L., Liu, R., Zhang, Y., Ji, Q., Zhang, H., et al. (2018). The effect of exposure to high altitude and low oxygen on intestinal microbial communities in mice. PLoS One 13:e0203701. doi: 10.1371/journal.pone.0203701

Conflict of Interest: The authors declare that the research was conducted in the absence of any commercial or financial relationships that could be construed as a potential conflict of interest.

Copyright (c) 2020 Li, Zhang, zhao, Wang, Wang and Wei. This is an open-access article distributed under the terms of the Creative Commons Attribution License (CC BY). The use, distribution or reproduction in other forums is permitted, provided the original author(s) and the copyright owner(s) are credited and that the original publication in this journal is cited, in accordance with accepted academic practice. No use, distribution or reproduction is permitted which does not comply with these terms. 